\title{
Characterization and optimization of the power consumption in wireless access networks by taking daily traffic variations into account
}

\author{
Margot Deruyck ${ }^{*}$, Emmeric Tanghe, Wout Joseph and Luc Martens
}

\begin{abstract}
In this study, a power consumption model as a function of the traffic is developed for macrocell base stations based on measurements on an actual base station. This model allows us to develop energy-efficient wireless access networks by combining the Green radio access network design (GRAND) tool designed by the authors, which develops an always-on network with a minimal power consumption for a predefined area, and an algorithm that introduces power reducing techniques in the network such as sleep modes and cell zooming. Green-field deployments and optimization of existing networks are investigated. For a green-field deployment, it was found that introducing sleep modes and cell zooming in the network can reduce the power consumption by up to $14.4 \%$ compared to the network without sleep modes and cell zooming. Optimizing existing networks by applying GRAND (without sleep modes and cell zooming) results in a power consumption reduction of 34.5\% compared to the original network. A careful selection of base station locations already results in a significant energy saving. Introducing sleep modes and cell zooming to the current networks results in a saving of 8\%. Sleep modes and cell zooming are promising energy-saving techniques for future wireless networks.
\end{abstract}

\section{Introduction}

Several studies indicate that within telecommunication networks, the wireless access networks are high power consumers [1-3]. Especially the base stations (BSs) are responsible for a significant part of the power consumption caused by wireless access networks. Therefore, a lot of effort has been put lately in designing new power reducing techniques such as sleep modes and cell zooming [4-12]. Sleep modes allow that a (part of the) BS can be switched off when there is no or little activity taking part in its coverage cell. Whenever necessary, the BS is waken up. When applying cell zooming, the cell size is adjusted adaptively according to the level of activity in a BS's cell. These techniques on their own can significantly reduce the power consumption in wireless access networks and combining them allows even higher power savings. Up to now

*Correspondence: margot.deruyck@intec.UGent.be

IBBT, Department of Information Technology, Ghent University, Gaston Crommenlaan 8 box 201, B-9050 Ghent, Belgium temporal variations of wireless access networks have only been studied in [7-12] and not experimentally.

In this study, a power consumption model as a function of the traffic load is developed for a macrocell BS based on measurements on an actual HSPA macrocell BS. To the best of author's knowledge, this has never been done before. Furthermore, this model is used in the Green radio access network design (GRAND) tool [13] to design an always-on network with a minimal power consumption for a predefined area. This deployment tool is then combined with an algorithm that introduces power reducing techniques in the network such as sleep modes and cell zooming. Both green-field deployments (i.e., implementing a new network) and optimizing an existing operator network by applying the GRAND tool are considered. To the best of the authors' knowledge, combining the power consumption model with the deployment tool and the algorithm is also a novelty.

\section{至 Springer}

(C) 2012 Deruyck et al.; licensee Springer. This is an Open Access article distributed under the terms of the Creative Commons Attribution License (http://creativecommons.org/licenses/by/2.0), which permits unrestricted use, distribution, and reproduction in any medium, provided the original work is properly cited. 
The outline of this article is as follows. Section 'Related study' gives an overview of the related study in this field. Section 'Load-dependent power consumption model for a macrocell base station' discusses the power consumption model of a macrocell BS. In Section 'Measurement procedure', the measurement procedure is described. In Section 'Relating power consumption and the number of voice and data calls', a model is given for the relation between traffic load and power consumption based on measurements. Section 'Introduction of sleep modes and cell zooming' describes the algorithm which determines which BSs can sleep and which have to 'zoom'. Section 'Results' presents the results of applying GRAND and the algorithm for introduction of sleep modes and cell zooming on a green-field deployment and on an existing operator network. In Section 'Conclusion', the conclusions of this study are given.

\section{Related study}

Recently, more attention is drawn to the power consumption in wireless access networks. To model this power consumption, it is important to quantify the power consumption of the different components in this network. [4-7] propose a power consumption model for today's macrocell BSs. However, [7] does not use any measurements to establish the power consumption model. The models proposed in [4-6] are based on GSM and/or UMTS macrocell BSs, while the model in this study is based on measurements on a HSPA BS. Our study shows that it is important to perform measurements to identify the relation between power consumption and traffic properly. Furthermore, a realistic HSPA traffic model can be determined instead of using theoretical traffic models as is often done in literature.

Also the possibilities of sleep modes to reduce the power consumption in wireless access networks is already established in a number of studies [7-10]. These studies discuss how sleep modes can be implemented and supported by the network. However, in [7-9], the energy savings in a realistic network deployment are not investigated. Our study tries to determine how much power can be saved by introducing sleep modes in a realistic network. In [10], the effect of sleep modes for different operator networks in a certain area is investigated which is a similar study as ours. However, it is also important to investigate what the energy savings are when sleep modes are introduced in a network with a minimal power consumption (here resulting from the GRAND tool). In this way, the most energy-efficient solution for the area is considered. The algorithm developed in [10] is similar to ours, but it is assumed that the cell size of the active (non-sleeping) BSs is maximized. In our algorithm, the cell size of the active BSs is expanded as much as needed which does not necessarily correspond with the maximum cell size. Note that in this study it is not discussed how sleep modes and cell zooming should be supported by the hardware, nor the protocols that will be needed, e.g., for waking up the BS when necessary. This is the scope of some other studies [8-12].

\section{Load-dependent power consumption model for a macrocell base station}

To optimize the energy efficiency of the wireless access network, it is important to determine the power consumption of the individual parts of this network. Here, the focus is on the macrocell BS. A typical architecture of this BS is shown in Figure 1. As discussed in [13], a macrocell BS consists thus of different components each consuming a specific amount of power. These components can be divided into two categories, the load independent and the load dependent (Equation (1)). Furthermore, two groups of equipment-one for all sectors and one per sector-can be unidentified (Equations (2) and(3)).

The first group contains all the components that are common for all sectors: the microwave link (that is used for communicating with the backhaul network and is, nowadays, often replaced by a fiber link) and the air conditioning. The power consumption of this equipment is constant throughout time. Although, a remark should be made. The temperature in the BS cabin should be kept more or less the same to preserve good functionality of the equipment. It is assumed that the heat generation rate (from both the external temperature of the cabin and the heat generated from the equipment) is constant which results in a constant power consumption of the air conditioning throughout time.

The second group is the equipment that is used per sector such as the rectifier (also known as the AC-DC

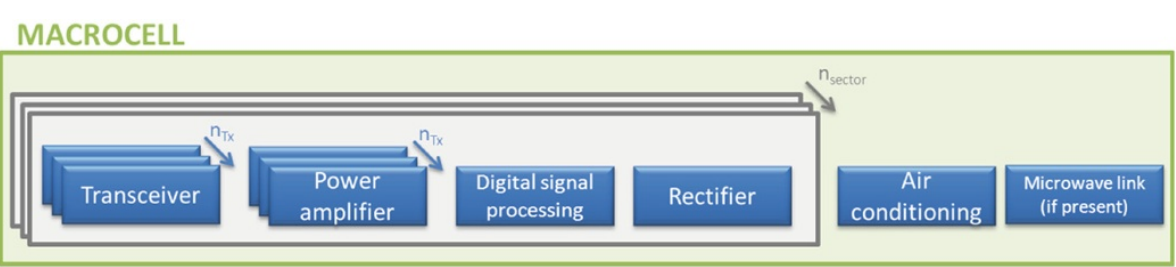

Figure 1 The architecture of a macrocell base station. 
converter), the digital signal processing (used for system processing and coding), the power amplifier, and the transceiver (for sending and receiving of the signals). The power consumption of this group has to be multiplied by the number of sectors $n_{\text {sectors }}$ supported by the BS. For a macrocell BS, $n_{\text {sectors }}$ is typically 3 .

Furthermore, the power consumption of these components (except for the rectifier) depends on the load on the BS which is determined by the number of users and the services they use in the BS's cell. The higher the number of users and the higher the requirement, the higher the load is. The power consumption of these components should thus be scaled according to the load. This is done by introducing a factor $F$, denoted here as the load factor.

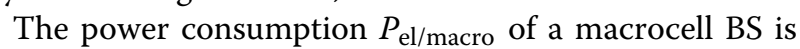
then determined as follows (in Watt):

$$
\begin{aligned}
& P_{\mathrm{el} / \mathrm{macro}}=P_{\mathrm{el} / \text { const }}+F \cdot P_{\mathrm{el} / \mathrm{load}} \\
& P_{\mathrm{el} / \mathrm{const}}=n_{\mathrm{sector}} \cdot P_{\mathrm{el} / \mathrm{rect}}+P_{\mathrm{el} / \text { link }}+P_{\mathrm{el} / \text { airco }} \\
& P_{\mathrm{el} / \mathrm{load}}=n_{\mathrm{sector}} \cdot\left(n_{\mathrm{Tx}} \cdot\left(P_{\mathrm{el} / \mathrm{amp}}+P_{\mathrm{el} / \text { trans }}+P_{\mathrm{el} / \mathrm{proc}}\right)\right)
\end{aligned}
$$

with $F$ the load factor and $n_{\text {sector }}$ the number of sectors supported by the BS, $n_{\mathrm{Tx}}$ the number of transmitting antennas per sector, $P_{\mathrm{el} / \text { rect }}, P_{\mathrm{el} / \text { link }}, P_{\mathrm{el} / \text { airco }}, P_{\mathrm{el} / \mathrm{amp}}$, $P_{\mathrm{el} / \text { trans }}, P_{\mathrm{el} / \mathrm{proc}}$ the power consumption of, respectively, the rectifier, the microwave link (if present), the air conditioning, the power amplifier, the transceiver and the digital signal processing (in Watt). The typical power consumption of the different components can be found in Table 1 [13]. The power consumption of the power amplifier is defined as follows (in Watt):

$$
P_{\mathrm{el} / \mathrm{amp}}=\frac{P_{\mathrm{Tx}}}{\eta}
$$

with $P_{\text {Tx }}$ the input power of the antenna (in Watt) and $\eta$ the efficiency of the power amplifier which is defined as the ratio of the RF (Radio Frequency) output power to the electrical input power [14].

In general, the load factor $F$ varies from 0 to 1 . A value of 0 indicates that the load-dependent components are consuming no power, i.e., there are switched off, while a value of 1 indicates that they are working on the highest power consumption level. Instead of determining a load factor $F$ for each hour of the day, a formula is defined to calculate $F$ as a function of the load caused by voice calls and the load caused by data calls. The formula will be of the following form:

$$
F=x \cdot V+y \cdot D+c
$$

with $V$ the load caused by voice calls $(0 \leq V \leq 1)$ and $D$ the load caused by data calls $(0 \leq D \leq 1)$. The division of load caused by voice calls and data calls is based on the traffic data received from a mobile operator. The parameters $x, y$, and $c$ will be determined based on measurements of both the power consumption and the voice and data traffic on an actual macrocell BS as discussed in the following sections. These measurements are necessary to relate the traffic data of the operator to the actual power consumption. These variations in power consumption can not be found in data sheets as they mostly provide the maximum or average power consumption.

\section{Measurement procedure}

As mentioned above, to determine a model for the load factor $F$, measurements are performed on an actual HSPA (High Speed Packet Access) macrocell BS in the suburban area of Ghent, Belgium. The power consumption of the BS is measured during six days (4 weekdays and

Table 1 Power consumption of the macrocell base station components

\begin{tabular}{lcc}
\hline Equipment & & $\begin{array}{c}\text { Macrocell } \\
\text { base station }\end{array}$ \\
\hline Number of sectors & $n_{\text {sector }}$ & 3 \\
Number of transmitting & $n_{\mathrm{Tx}}$ & 1 \\
antennas per sector & $P_{\mathrm{el} / \text { proc }}$ & $100 \mathrm{~W}$ \\
Digital signal processing & $\eta$ & $12.8 \%$ \\
Power amplifier & $P_{\mathrm{el} / \text { trans }}$ & $100 \mathrm{~W}$ \\
Transceiver & $P_{\mathrm{el} / \text { rect }}$ & $100 \mathrm{~W}$ \\
Rectifier & $P_{\mathrm{el} / \text { airco }}$ & $225 \mathrm{~W}$ \\
Air conditioning & $P_{\mathrm{el} / \text { ink }}$ & $80 \mathrm{~W}$ \\
Microwave link & $P_{\mathrm{el} / \mathrm{const}}$ & $605 \mathrm{~W}$ \\
& $P_{\mathrm{el} / \text { load }}$ & $1067.7 \mathrm{~W}$ \\
\hline Total BS $(\mathbf{F}=\mathbf{0})$ & $\boldsymbol{P}_{\mathrm{el} / \text { macro }}$ & $\mathbf{6 0 5} \mathbf{W}$ \\
Total BS $(\mathbf{F}=\mathbf{1})$ & $\boldsymbol{P}_{\mathrm{el} / \text { macro }}$ & $\mathbf{1 6 7 2 . 7} \mathbf{W}$ \\
\hline
\end{tabular}


2 weekend days). Only the power consumption of the load-dependent components (i.e., the digital signal processing, the transceiver, and the power amplifier) were included in these measurements. As DC (Direct Current) is used in the BS, the voltage is constant (i.e., approximately $54 \mathrm{~V}$ ) and thus only the current was measured. The power consumption $P(t)$ at a certain time instance $t$ is then determined as follows (in Watt):

$$
P(t)=V \cdot I(t)
$$

with $V$ the voltage (in Volt) and $I(t)$ the current at time $t$ (in Ampere).

Using an AC/DC current clamp (Fluke i410), the current is measured every second which results in 518,400 samples for the measurement period of six days.

\section{Relating power consumption and the number of voice and data calls \\ Processing the measurement data}

Based on the data from the measurements, a model for the load factor $F$ is determined. The following procedure is used. First, the average power consumption for each hour during the measurement period is determined. This gives us a vector $\mathbf{P}$ containing 144 ( $=6$ days $\times 24 \mathrm{~h}$ ) power consumption values, i.e., for each hour of the measurement period one value. This averaging is necessary as the power consumption will later be related to the number of voice and data calls. The data about the number of voice and data calls is provided per hour by the operator.

Second, the averaged power consumption per hour $\mathbf{P}$ is normalized [15]:

$$
\mathbf{P}_{\text {norm }}=\frac{\mathbf{P}-\min (\mathbf{P})}{\max (\mathbf{P})-\min (\mathbf{P})}
$$

with $\mathbf{P}$ the vector of the average power consumption per hour, and $\max (\mathbf{P})$ and $\min (\mathbf{P})$ the maximum, respectively minimum, average power consumption per hour during the measurement period. $\mathbf{P}_{\text {norm }}$ is thus a vector of again 144 values, which represent the normalized power consumption per hour during the measurement period. $\mathbf{P}_{\text {norm }}$ is dimensionless and yields values between 0 and 1 . The data is normalized because the relative values are more interesting than the absolute values.

Figure 2 shows $\mathbf{P}_{\text {norm }}$ during one of the measurement days for the considered HSPA macrocell BSs. $\mathbf{P}_{\text {norm }}$ equals $F$, as $F$ is defined as a scale factor taking the influence of the traffic on the power consumption into account. From 6 a.m. on, $F$ increases significantly as during the day more people make phone calls than during the night. A higher value of $F$ will result in a higher power consumption as can be seen from Equation (1). In the evening, the load factor $F$ decreases again. Around 7 p.m., a small peak of demand can be noted. Similar results are found for the other measurement days.
As mentioned above, $\mathbf{P}_{\text {norm }}$ will be related to the HSPA traffic.Two categories of traffic can be defined: voice calls and data calls. The data about the number of voice and data calls is provided per hour by the mobile operator. The vectors $\mathbf{V}_{\mathbf{h}}$ and $\mathbf{D}_{\mathbf{h}}$ contain thus 144 ( $=6$ days $\times 24 \mathrm{~h}$ ) values which represent the number of voice, respectively data, calls for each hour $h$ of the measurement period. The same procedure as for the power consumption is then followed for both the number of voice and data calls and thus the number of voice and data calls are normalized:

$$
\begin{aligned}
\mathbf{V}_{\text {norm }} & =\frac{\mathbf{V}_{\mathbf{h}}-\min \left(\mathbf{V}_{\mathbf{h}}\right)}{\max \left(\mathbf{V}_{\mathbf{h}}\right)-\min \left(\mathbf{V}_{\mathbf{h}}\right)} \\
\mathbf{D}_{\text {norm }} & =\frac{\mathbf{D}_{\mathbf{h}}-\min \left(\mathbf{D}_{\mathbf{h}}\right)}{\max \left(\mathbf{D}_{\mathbf{h}}\right)-\min \left(\mathbf{D}_{\mathbf{h}}\right)}
\end{aligned}
$$

with $\mathbf{V}_{\mathbf{h}}$ and $\mathbf{D}_{\mathbf{h}}$ the vectors containing the number of voice, respectively data, calls during each hour of the measurement period, $\max \left(\mathbf{V}_{\mathbf{h}}\right)$ and $\max \left(\mathbf{D}_{\mathbf{h}}\right)$ the maximum number of voice, respectively data, calls per hour during the whole measurement period and $\min \left(\mathbf{V}_{\mathbf{h}}\right)$ and $\min \left(\mathbf{D}_{\mathbf{h}}\right)$ the minimum number of voice, respectively data, calls per hour during the whole measurement period. $\mathbf{V}_{\text {norm }}$ and $\mathbf{D}_{\text {norm }}$ are then vectors containing 144 values representing the normalized number of voice, respectively data, calls for each hour of the measurement period. The values of these vectors vary from 0 to 1 .

Figure 2 compares $\mathbf{V}_{\text {norm }}$ and $\mathbf{D}_{\text {norm }}$ with the load factor and normalized power consumption $\mathbf{P}_{\text {norm }}$ for one of the measurement days of the considered HSPA macrocell BS. A similar trend as for $F$ can be noticed. The number of voice and data calls are also higher during daytime. So $F$ and $V$ and $D$ will be correlated and modeled in the following section.

\section{Determining a model for the load factor $F$}

Based on the results shown in Figure 2, a model for the load factor $F$ is now determined by using linear regression. The dependent variable is $\mathbf{P}_{\text {norm }}$, while the two independent variables are $\mathbf{V}_{\text {norm }}$ and $\mathbf{D}_{\text {norm }}$. The following formula is then obtained for $F$ :

$$
F=x \cdot V+y \cdot D+z \cdot V \cdot D+c
$$

wit $V$ equals $V_{\text {norm }}$ and $D$ equals $D_{\text {norm }}$. As $y$ and $z$ are not significant ( $p$-value $=0.7128$ and 0.5044 , respectively), Equation (10) can be adapted to:

$$
\begin{aligned}
F & =x \cdot V+c \\
& =0.6 \cdot V+0.2
\end{aligned}
$$

with $x=0.6$ and $c=0.2$. The model is shown in Figure 2 as a function of the time and shows very good agreement with the measured data.

As can be seen from the form of Equation (12), the BS's power consumption depends linearly on $V$. In [5,6], a similar power consumption model is proposed 


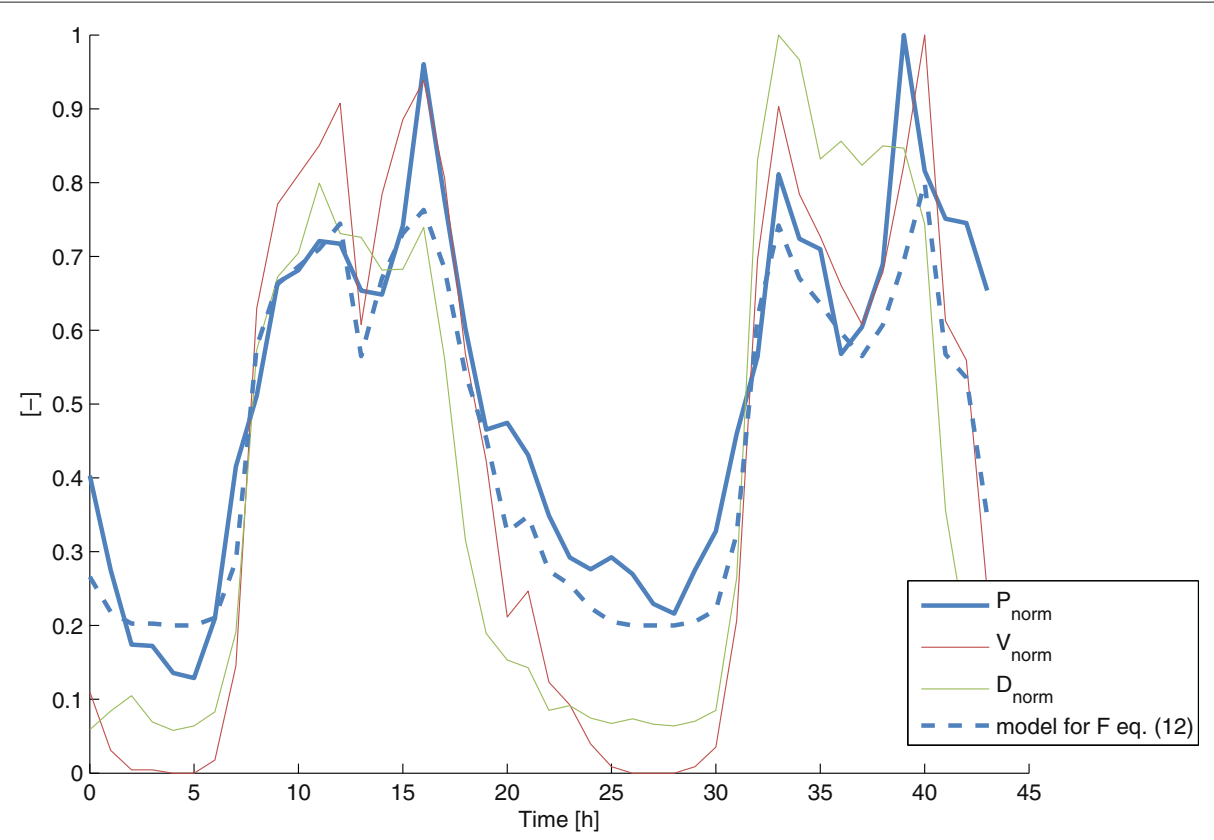

Figure 2 Comparison of $\mathrm{P}_{\text {norm }}, \mathrm{V}_{\text {norm }}$, and $\mathrm{D}_{\text {norm }}$ as a function of time during $24 \mathrm{~h}$ for a HSPA macrocell base station (suburban environment, Belgium).

based on measurements on a GSM and UMTS macrocell BSs. Both these studies also obtain a linear relation between the traffic and the power consumption which validates our model. The daily power consumption increases with $1.5 \%$ when the power consumption during sleep mode increases with $100 \mathrm{~W}$. Note that when $V$ equals to 0 , the power consumption does not decrease to $0 \mathrm{~W}$ because the load-independent components (such as, the rectifier, the air conditioning, and the microwave link) still consume power and also the load-dependent components keep consuming a small amount of power $(F=0.2$ for $V=0)$.

Note also that the parameters $(x, y, z, c)$ depend on the performance of the components. A component of another brand or another model number can result in different parameters $(x, y, z, c)$. The values of Table 1 are average values obtained from data sheets from various vendors. The BS's power consumption calculated using these values agrees excellently (standard deviation of $2 \%$ ) with the measured ones, presented in this article. The obtained values for $(x, y, z, c)$ are representative for the current generation BSs. For new generation BSs and future developments where the power consumption of the components is optimized, new measurements will be necessary.

\section{Introduction of sleep modes and cell zooming}

A promising technique to reduce power consumption in wireless access networks is the introduction of sleep modes where BSs are becoming inactive when no or little activity takes place in their coverage cells [7-10]. The BS is not completely switched off during the sleep mode as it keeps monitoring and if necessary (e.g., when a call has to be made) it can become active again. Another technique is called cell zooming [11] which adaptively adjust the cell size according to (amongst others) the traffic load. In this section, the designed algorithm, which combines these two techniques for power consumption reduction in a wireless access network, is discussed.

The algorithm is discussed based on Figure 3. Figure 3a shows the initial situation, i.e., the always-on network as obtained from the GRAND tool. For each BS in the network, it is checked if it is possible to put it into sleep. However, when a BS is put into sleep the coverage of this BS should be guaranteed by other BS(s), which are awake. To define if this is possible for a certain BS, here called $\mathrm{BS}_{\text {test }}$, the neighboring BSs are determined as shown in Figure 3a. The range of the neighboring BSs will then be increased by adding up to $5 \mathrm{dBm}$ to the antenna's input power of the neighboring BSs which is shown by the arrows in Figure $3 \mathrm{~b}$. If the area covered by $\mathrm{BS}_{\text {test }}$ can be covered by extending the range of neighboring $\mathrm{BS}(\mathrm{s})$, $\mathrm{BS}_{\text {test }}$ will be put into sleep when the sleep threshold is met as shown in Figure 3c. The dotted line in this figure indicates the 'old' coverage of the neighboring BSs. The traffic load of $\mathrm{BS}_{\text {test }}$ will be divided over the neighboring BSs. When the sleep threshold is not met, the final 

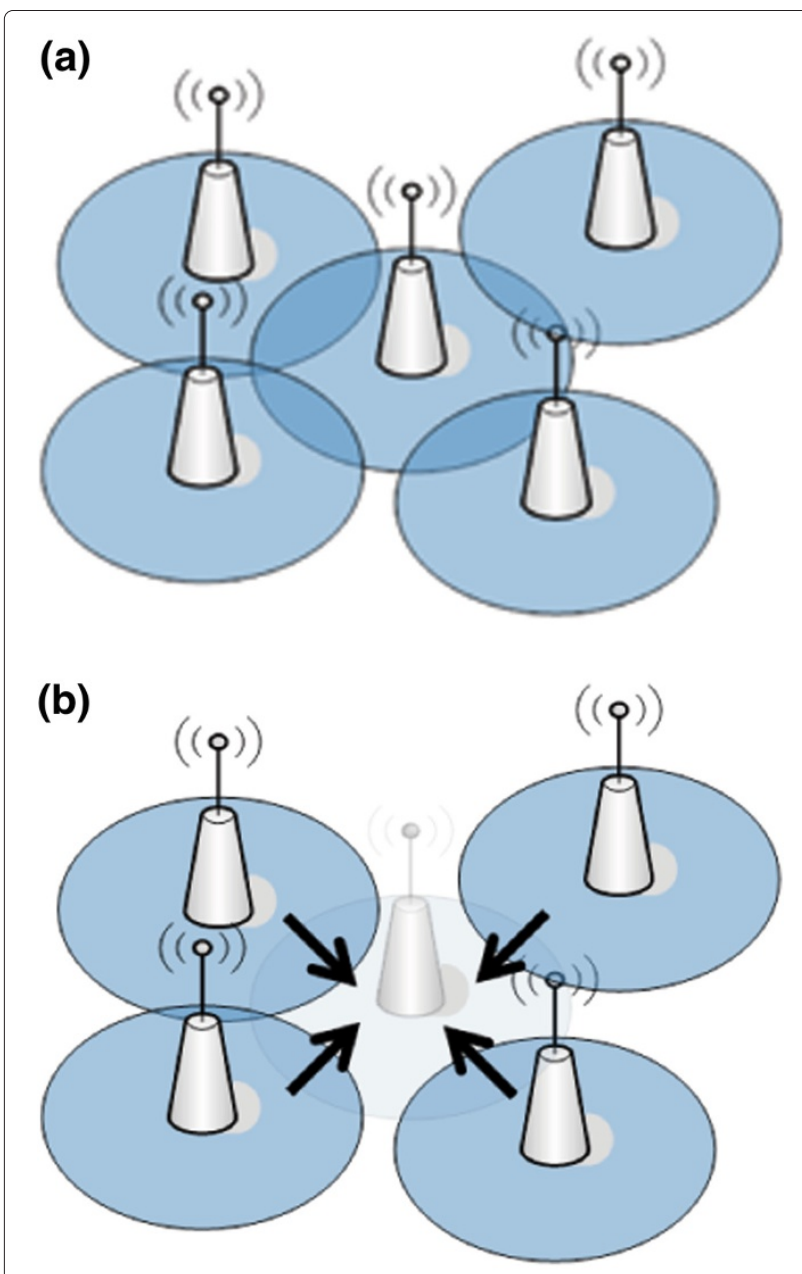

(c)

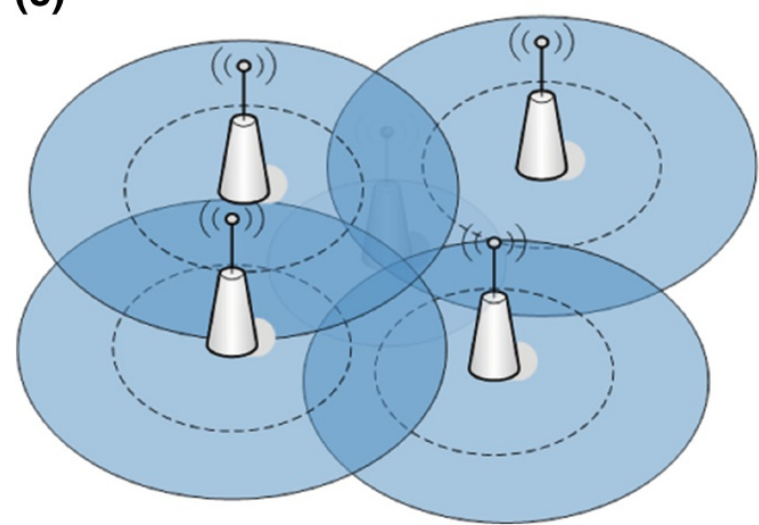

Figure 3 Overview of the proposed algorithm for introducing sleep modes and cell zooming: always-on configuration (a), checking if a base station can sleep (b), final configuration with base station asleep (c).

configuration is as shown in Figure 3a. When the sleep threshold is met, the final configuration is as shown in Figure 3c.
As discussed above, the algorithm consists of a number of steps. The different steps of the algorithm will now be discussed in details based on the flow graph in Figure 4.

First, a network is designed for a predefined area by using the GRAND tool [13]. This tool can be used to design greenfield networks, but also enables optimizing existing networks. The input power of the (macrocell) BS antenna is limited in this first step to e.g., the maximum possible input power minus $5 \mathrm{dBm}$. In this way, it is possible to let cells zoom by adjusting the input power of the antenna.

Second, the algorithm for introduction of sleep modes and cell zooming is applied (Figure 4). The different steps are now explained. For every active base station $\mathrm{BS}_{i}(i=1, \ldots, M$ with $\mathrm{M}$ the total number of $\mathrm{BSs})$ in the network resulting from the GRAND tool, it is determined whether it is possible to put this BS to sleep. This is done by selecting the 4 closest active BSs $\left(\mathrm{BS}_{j}\right)_{i}$ $\left(j=1, \ldots, 4\right.$ with $j=1$ the BS the closest to $\mathrm{BS}_{i}$ and $j=4$ the BS the furthest to $\mathrm{BS}_{i}$ ) (Figure 4 Step 1). Remark that the number of closest BSs can be chosen freely. Here, 4 was chosen as this gave a good balance between performance and the computational time of the algorithm.

In the next steps, the input power of the antenna of one or more neighboring BSs is increased. This is done as follows:

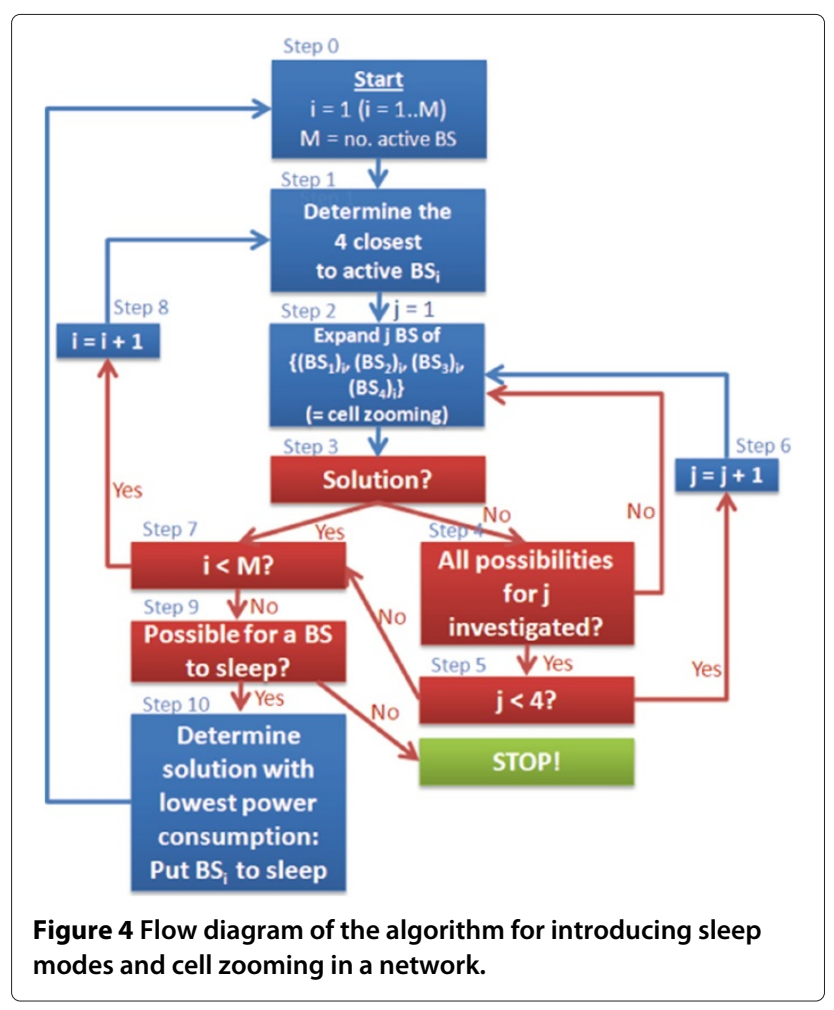


1. Increase the input power of the antenna of $\left(\mathrm{BS}_{1}\right)_{i}$ with $1 \mathrm{dBm}$, i.e., apply cell zooming (Figure 4 Step 2). This will expand the range of the cell. Note that it is important to increase the input power sequential because each $\mathrm{dBm}$ added to the input power results in a higher power consumption as shown in Equations (3) and (4).

2. Check if a solution is found. This means: check if the cell of $\left(\mathrm{BS}_{1}\right)_{i}$ covers the cell of $\mathrm{BS}_{i}$ (Figure 4 Step 3). If so, the calculation for $\mathrm{BS}_{i}$ can be stopped (Figure 4 Step 8).

3. If not, follow the same procedure (step 1 and 2 of this list) for all the other BSs from set $\left(\mathrm{BS}_{j}\right)_{i}($ Figure 4 Step 4).

4. If no solution can be found by expanding the range of only one $\mathrm{BS}$ from the set $\left(\mathrm{BS}_{j}\right)_{i}$, check if there is solution when expanding the range of two BSs from $\left(\mathrm{BS}_{j}\right)_{i}$ (Figure 4 Step $5 \& 6$ ). If a solution is found, stop the calculation for $\mathrm{BS}_{i}$ (Figure 4 Step 3).

5. If all combinations with two BSs from set $\left(\mathrm{BS}_{j}\right)_{i}$ are checked and no solution is found (Figure 4 Step 5 \& 6), check if there is a solution when expanding the range of three $\mathrm{BSs}$ from $\left(\mathrm{BS}_{j}\right)_{i}$ (Figure 4 Step 5 \& 6). If a solution is found, stop the calculation for $\mathrm{BS}_{i}$ (Figure 4 Step 3).

6. If all combinations with three $\mathrm{BSs}$ from set $\left(\mathrm{BS}_{j}\right)_{i}$ are checked and no solution is found (Figure 4 Step 5 \& 6), check if there is a solution when expanding the range of the four BSs from $\left(\mathrm{BS}_{j}\right)_{i}$. If a solution is found, stop the calculation for $\mathrm{BS}_{i}$ (Figure 4 Step 3).

7. If no solution can be found by expanding the range of the four BSs from the set $\left(\mathrm{BS}_{j}\right)_{i}$, it is not possible to put the BS into sleep.

When a solution is found for $\mathrm{BS}_{i}$ or when no solution is found at all for $\mathrm{BS}_{i}$, it is checked if there are other active BSs in the network which have not been investigated yet (Figure 4 Step $7 \& 8$ ). If so, the above described procedure is repeated for these BSs. If not, it is checked if there was a solution for any of the active BSs in the network (Figure 4 Step 9). If so, the solution with the lowest power consumption is determined and the corresponding base station $\mathrm{BS}_{i}$ is put into sleep (Figure 4 Step 10). To determine the power consumption of a solution, the traffic load of the BS put into sleep mode is equally divided over the BSs that need to be expanded. This is a very simple approach, but it is expected that more complex approaches will not change the results as the power consumption depends linearly on the number of voice calls $V$ (see Equation (12)). The whole procedure is then again repeated (Figure 4 Step 0). If no solution is found for any of the active BSs $\mathrm{BS}_{i}$ in the network (Figure 4 Step 9), the algorithm is stopped and the final result is obtained.

\section{Results}

In this section, the power consumption of the macrocell BS is determined as a function of the load $V$ caused by voice calls. Furthermore, the evolution of the BS's power consumption during the day is investigated.

The evolution of the macrocell BS's power consumption during the day is shown in Figure 5. This figure is obtained by taken the average of the power consumption over the measurement period for each hour of the day. The highest power consumption (i.e., $1259.2 \mathrm{~W}, V=0.7$ ) is obtained at 5 p.m., while the lowest power consumption (i.e., $820.4 \mathrm{~W}$, $V=0.003$ ) is reached at $4 \mathrm{a} . \mathrm{m}$. The power consumption is clearly higher during daytime because less people make phone calls at night (Figure 5).

\section{Green-field deployment}

The power savings in a network by introducing sleep modes and cell zooming are now determined for a greenfield deployment. For this investigation, a new network is developed for the city center of Ghent. This target area is shown in Figure 6a and has a surface of approximately $13.3 \mathrm{~km}^{2}$. A coverage requirement of $90 \%$ of the target area is here assumed. Furthermore, LTE (Long Term Evolution) is chosen as wireless technology (frequency of $2.6 \mathrm{GHz}$ ) [16] and a physical bit rate of approximately $5 \mathrm{Mbps}$ (in a $5 \mathrm{MHz}$ channel) is provided. LTE is here considered as it is assumed that this technology will mostly be introduced in new network deployments. It is assumed that the (voice) traffic behaviour will not change significantly when a new technology is introduced in the network. Comparing the shape of the daily evolution of the HSPA traffic with the shape of the daily evolution of GSM (Global System for Mobile communications) as shown in [15], shows no significant differences. Therefore, the model from the previous sections is also used here. Furthermore, it is assumed that the traffic is uniformly distributed over the area. Each BS will thus have the same traffic load at the same time when sleep modes are not activated.

The network resulting from the GRAND tool (without activation of sleep modes and cell zooming) is shown in Figure $6 \mathrm{~b}$. It consists of 80 macrocell BSs with a total power consumption of $1566 \mathrm{kWh}$ per day.

Figure $6 \mathrm{c}$ shows the resulting network when sleep modes and cell zooming are activated. 28 BSs can be put into sleep when the sleeping conditions are met (i.e., when $V$ is below the sleep threshold). Note that it is here not necessary to define the actual value of the sleep threshold, as it is assumed that each BS has the same traffic pattern, each BS would reach the sleep threshold at the same time, and thus it would be possible to put each BS into sleep. However, the coverage that is lost by putting a BS into sleep should be provided by another BS(s). The algorithm shown in Figure 4 determines for which BSs 


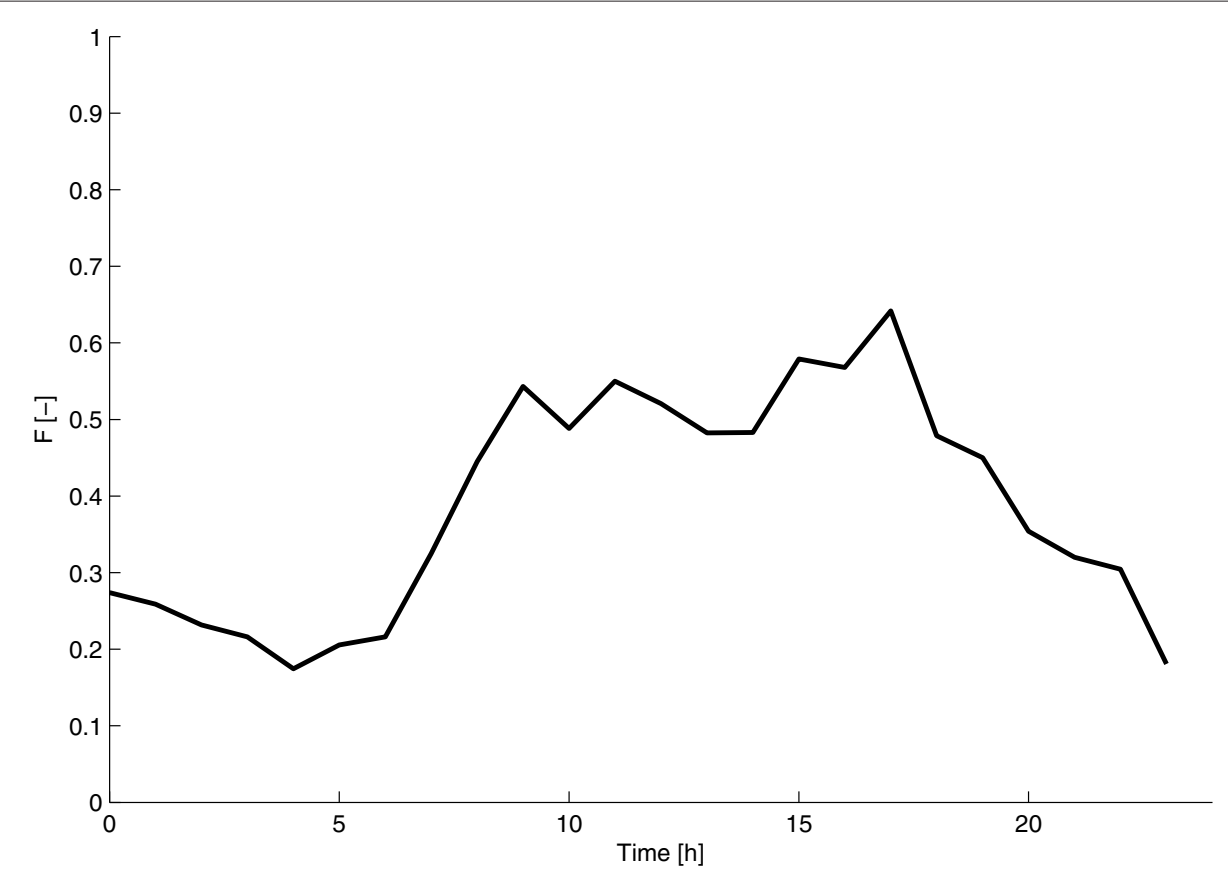

Figure 5 Evolution of $\boldsymbol{F}$ during the day.

this is possible and for which not. The sleep threshold will be a very important parameter in determining the actual power savings in the network as is now discussed.
Figure 7 shows the power savings that can be achieved by introducing sleep modes and cell zooming in the network. Here, we vary the sleep threshold from 0.0 to 0.3 in

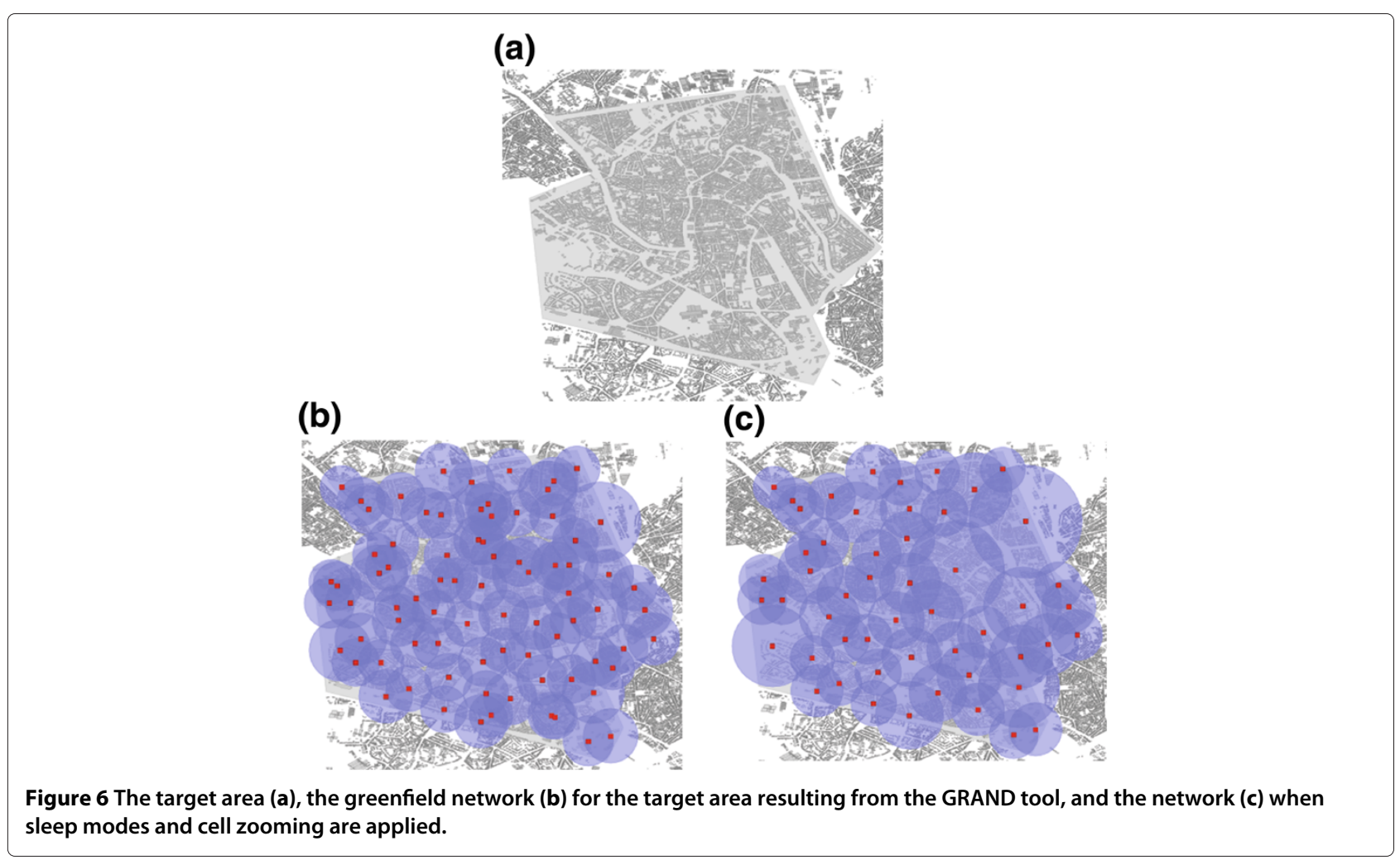




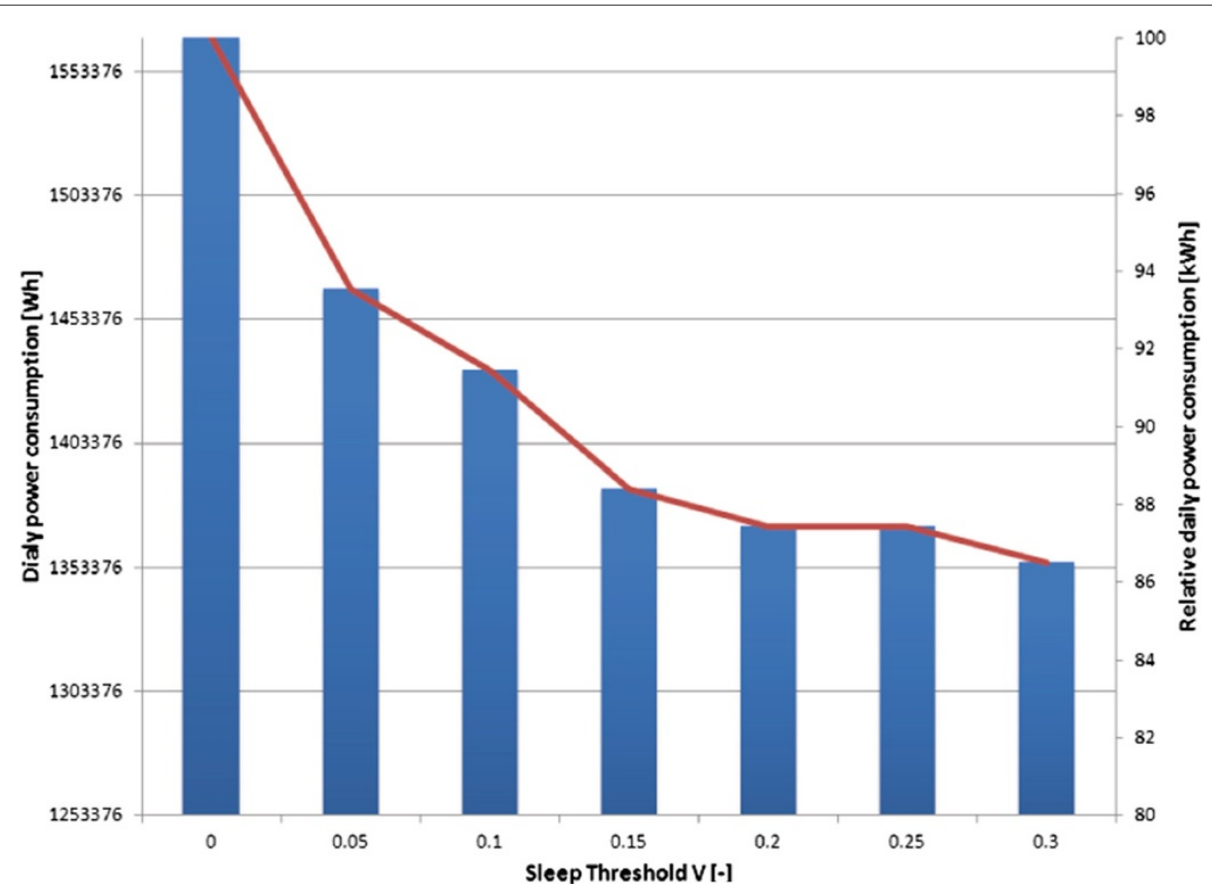

Figure 7 Power savings by introducing sleep modes and cell zooming in the network for varying sleep thresholds from 0 to 0.3 .

steps of 0.05 . The daily power consumption of the network as a function of the sleep threshold is shown in Figure 7. When the sleep threshold is 0.1 , the network consumes $1432.8 \mathrm{kWh}$ per day, which is $8.6 \%$ lower than without sleep modes and cell zooming. If the sleep threshold is 0.2 , the network only consumes $1369.9 \mathrm{kWh}$ per day which corresponds with a power saving of $12.6 \%$. If the sleep threshold is 0.3 , the network only consumes $1297.3 \mathrm{kWh}$ per day which corresponds with a power saving of $13.5 \%$. A higher power saving is achieved when a higher threshold is applied because the BSs can sleep more hours per day than for a lower threshold (Section 'Results'). However, for a sleep threshold of 0.3 , the load on the neighboring BSs is becoming very high (load of 0.9). Therefore, it is recommended to use a sleep threshold lower than 0.3. Furthermore, remark that it is assumed that a BS in sleep mode consumes no power (both the load dependent and the load independent power consumption equal $0 \mathrm{~W}$ ). This might however be too optimistic, but it allows us to determine the upper bounds of the power savings that can be achieved. Figure 8 shows the influence of this assumption on our results. The power consumption for the sleep mode is varied between $0 \mathrm{~W}$ and $600 \mathrm{~W}$ (which corresponds with the BS's power consumption when there is no traffic (Table 1)) and a sleep threshold of 0.1 is assumed. The percentages show the relative daily power consumption with respect to the daily power consumption of the network without sleep modes. A linear relation between the power consumption of the sleep mode and the daily power consumption of the network is found. As mentioned above, 28 BSs can put into sleep during 8 hours of the day (sleep threshold $=0.1$ ). If a power consumption of $100 \mathrm{~W}$ is assumed for the sleep mode, this results in an extra power consumption of $22.4 \mathrm{kWh}$. The higher the power consumption during sleep mode, the lower the obtained power savings. When a BS consumes $600 \mathrm{~W}$ during sleep modes, the network's power consumption is the same as for the network without sleep modes.

Figure 9 shows the evolution of the network's power consumption during a day with and without sleep modes. The power consumption during daytime (from 8 a.m. till 7 p.m.) is similar for all the investigated cases (i.e., no sleep modes and sleep modes with the considered sleep thresholds) due to the fact that for this time period $V$ is higher than the sleep thresholds considered. At night (1 a.m. -7 a.m.) and 11 p.m., 28 of the 80 BSs can be put into sleep ( $V$ is lower than all the sleep thresholds considered). For the other hours of the day, it depends on the sleep threshold if the $28 \mathrm{BSs}$ can be put into sleep.

\section{Optimizing a network}

For this investigation, Brussels Capital Region, Belgium, is considered with a surface of approximately $149 \mathrm{~km}^{2}$. Data about the BS sites is provided by BIPT (Belgian Institute for Postal services and Telecommunications). Figure 10a shows all the 858 available BS sites in Brussels. Within this region, four different operator networks can be identified. 


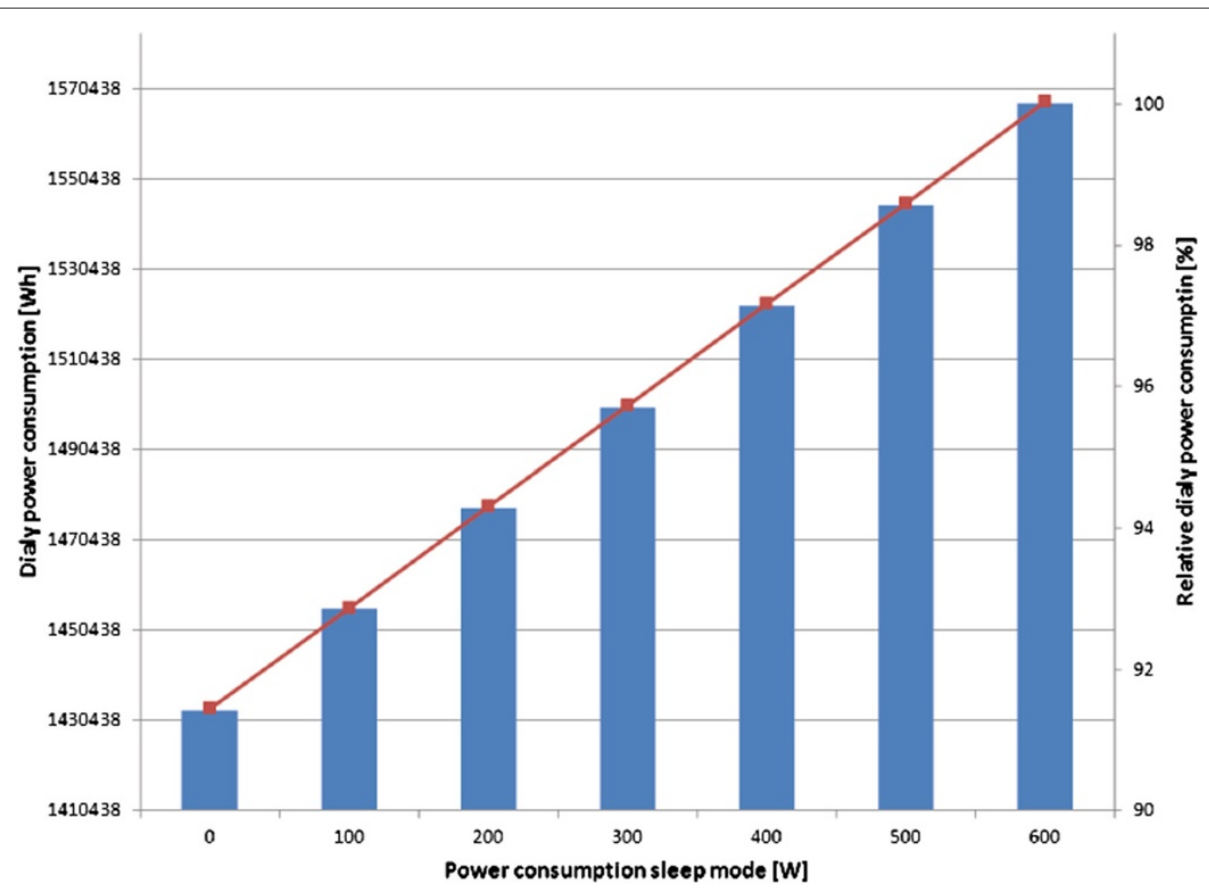

Figure 8 Influence of the power consumption during sleep mode on daily power consumption of the network (sleep threshold $=0.1$ ).

Figure 10a shows thus all the BSs of the four operators. Here, only one operator network (289 BSs, HSPA [17]) is considered. All the possible locations are shown in Figure 10b and are used as an input for the GRAND tool. The GRAND tool will adapt the operator network by adding BSs, changing the locations of BSs, or changing settings such as the input power of the antenna. By changing the locations of BSs, it is meant that a BS is removed from a location and added at another possible location. The set of BSs that can be added to the

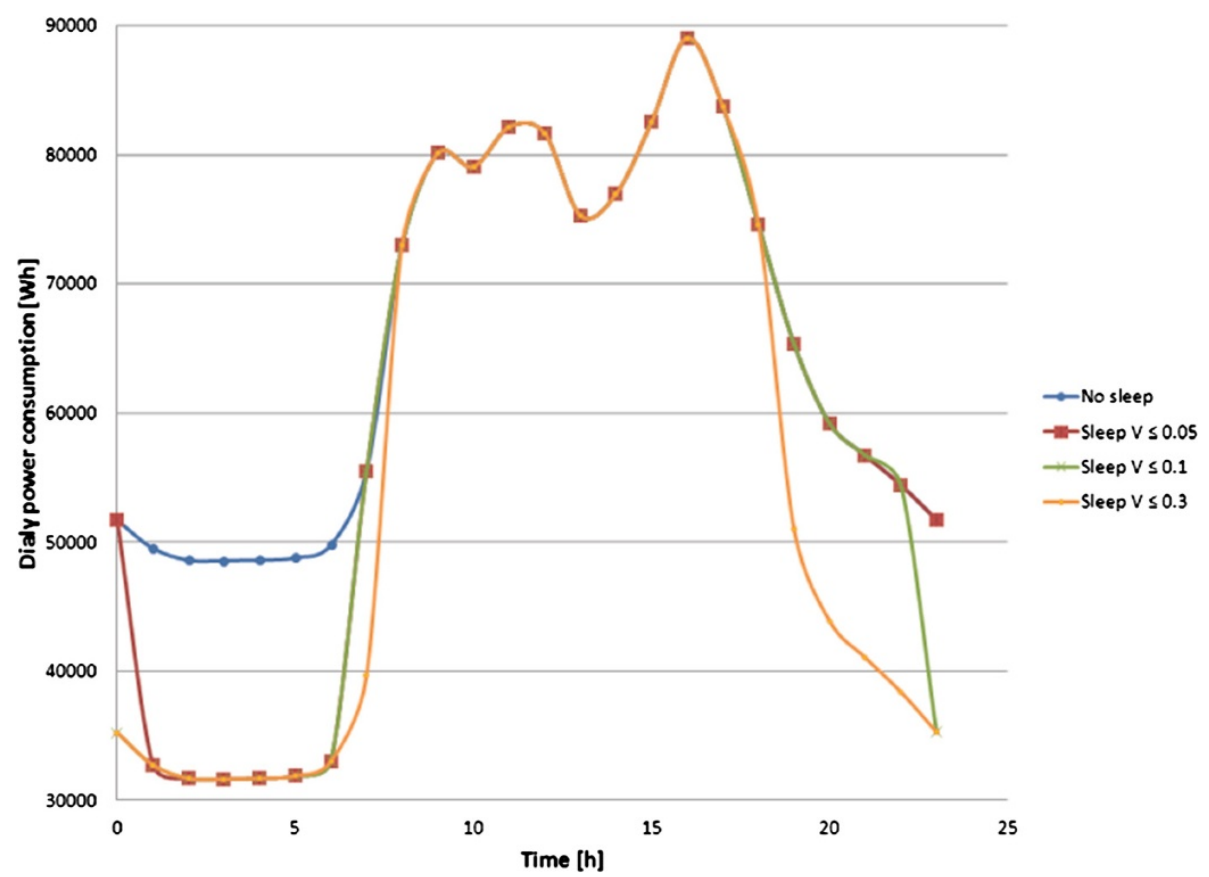

Figure 9 The evolution of power consumption of the network through time when sleep modes are activated and deactivated. 


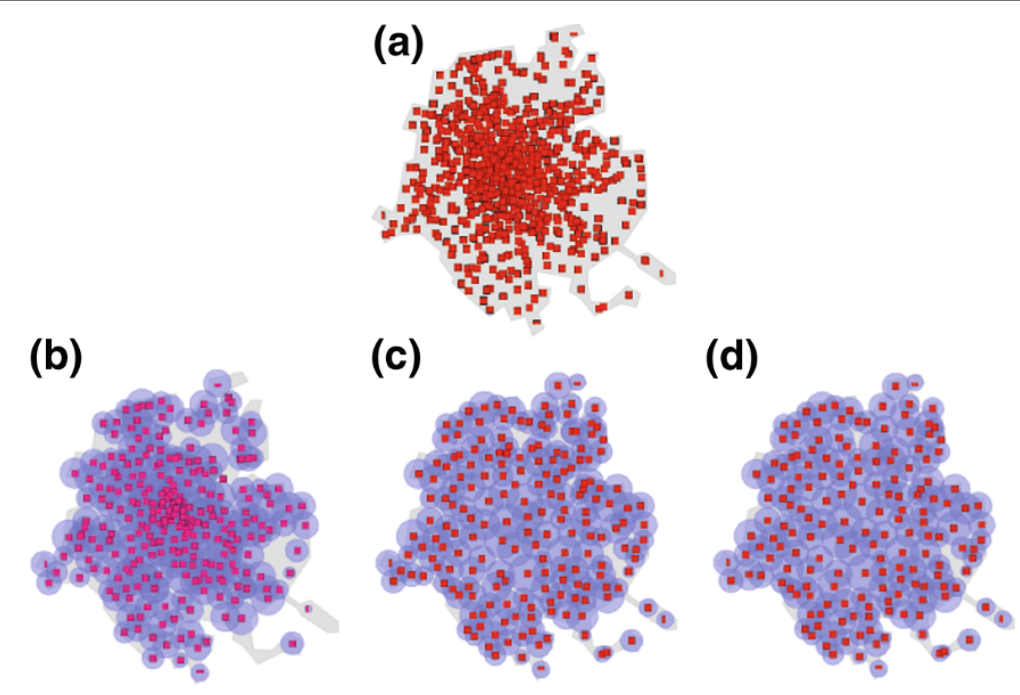

Figure 10 Brussels capital region: available sites (a), original operator network (b), network optimized by the GRAND tool (c), network with sleep modes and cell zooming activated.

network corresponds with the set of all the available BSs in the Brussels Capital Region (Figure 10a). Furthermore, it is assumed that the traffic is uniformly distributed over the considered area for both the existing and the optimized network. The model from the previous sections will be used in this study which is a realistic approach as only the HSPA macrocell BSs are considered for the existing network.

Figure 10c shows the network resulting from the GRAND tool with deactivation of the sleep modes and cell zooming. This network uses only 188 BSs and has thus a daily power consumption that is $33.4 \%$ less than the power consumption of the original network $(4733.4 \mathrm{kWh}$ versus $7108.0 \mathrm{kWh}$ ) as shown in Table 2 . Remark that the optimized network with its lower power consumption still has a significant higher coverage than the original network (95.0\% versus $88.7 \%$, Table 2). It is thus concluded that it is useful to place the BSs properly to save power.

Figure 10d shows the optimized network when sleep modes (sleep threshold of 0.1) and cell zooming are activated. 18 BSs can sleep when the traffic is below the sleep threshold of 0.1 (Table 2). This results in a daily power consumption of $4641.4 \mathrm{kWh}$ (Table 2) which is $1.9 \%$ lower than the optimized network without activating sleep modes and $33.4 \%$ lower than the original operator network. Again it is concluded that it is recommended to add sleep modes and cell zooming into the networks. Today's operator networks are currently thus not optimized towards power consumption.

For comparison, it is also determined how much power can be saved when introducing sleep modes in the original operator network without first optimizing the network. Assuming a sleep threshold of 0.1 , this results in a daily power consumption of $6526.5 \mathrm{kWh}$ (versus $7108.0 \mathrm{kWh}$ for the original network). A reduction of about $8 \%$ is thus obtained.

\section{Conclusion}

In this study, a power consumption model as a function of the traffic load and temporal variations is developed for a macrocell base station based on measurements on an actual base station. This model allows us todevelop energy-efficient wireless access networks by combining the GRAND tool, which develops an always-on network with a minimal power consumption for a predefined area, and an algorithm that introduces power reducing

Table 2 Results for the optimization of an operator network in the Brussels caption region

\begin{tabular}{|c|c|c|c|}
\hline & Original & $\begin{array}{l}\text { Optimized } \\
\text { no sleep }\end{array}$ & $\begin{array}{c}\text { Optimized } \\
\text { sleep }\end{array}$ \\
\hline No. base stations & 289 & 188 & 170 \\
\hline Coverage (\%) & 88.7 & 95.0 & 95.0 \\
\hline Daily power consumption (kWh) & 7108.0 & 4733.4 & 4641.4 \\
\hline
\end{tabular}


techniques in the network such as sleep modes and cell zooming.

Two cases are investigated. In the first case, a completely new LTE network is developed for the city center of Ghent. By introducing sleep modes and cell zooming in this network, the power consumption can be reduced up to $14.4 \%$ (depending on the used sleep threshold) compared to the network without sleep modes and cell zooming. In the second case, an existing operator network for Brussels capital region is optimized. Applying GRAND on this network results in a reduction of $34.5 \%$ for the daily power consumption. The introduction of sleep modes and cell zooming causes an additional saving of $2.5 \%$ compared to the optimized network. A careful selection of the base station locations can already result in a significant saving $(34.5 \%$ as shown by the results from the GRAND tool). In current networks, this can be done by site sharing. For future networks, it is recommended that sleep modes and cell zooming are supported.

Future research will consist of taking also adaptive capacity demands into account and exposure for the human being.

\section{Competing interests}

The authors declare that they have no competing interests.

\section{Acknowledgements}

The study described in this article was carried out with support of the IBBT-project Green ICT. W. Joseph was a Post-Doctoral Fellow of the FWO-V (Research Foundation Flanders).

Received: 6 January 2012 Accepted: 18 July 2012

Published: 8 August 2012

\section{References}

1. W Vereecken, W Van Heddeghem, M Deruyck, B Puype, B Lannoo, W Joseph, D Colle, L Martens, M Pickavet, Power consumption in telecommunication networks: overview and reduction strategies. IEEE Commun. Mag. 49(6), 62-69 (2011)

2. O Blume, D Zeller, U Barth, Approaches to energy efficient wireless access networks. in 4th International Symposium on Communications, Control and Signal Processing (ISCCP) (Limassol, Cyprus, 2010), pp. 1-5

3. M Pickavet, W Vereecken, S Demeyer, P Audenaert, B Vermeulen, C Develder, D Colle, B Dhoedt, P Demeester, Worldwide energy needs for $\mathrm{ICT}$ : the rise of power-aware networking. in 2nd International Symposium on Advanced Networks and Telecommunication Systems (ANTS) (Bombay, India, 2008), pp. 1-3

4. O Arnold, F Richter, G Fettweis, O Blume, Power consumption modeling of different base station types in heterogeneous cellular networks. in Future Network and Mobile Summit (Florence, Italy, 2010), pp. 1-8

5. C Peng, S-B Lee, S Lu, H Luo, H Li, Traffic-driven power savings in operational $3 \mathrm{G}$ cellular networks. in Proceedings of the 17th Annual Conference on Mobile Computing and Networking (MobiCom) (Las Vegas, Nevada, 2011) doi:10.1145/2030613.2030628

6. J Lorincz, T Garma, G Petrovic, Measurements and modelling of base station power consumption under real traffic loads. Sensors. 12(4), 4281-4310 (2012)

7. G Micallef, P Mogensen, $\mathrm{H}-\mathrm{O}$ Scheck, Cell size breathing and possibilities to introduce cell sleep mode. in 16th European Wireless conference 2010 (Lucca, Italy, 2010), pp. 111-115

8. L Correia, D Zeller, O Blume, D Ferling, Y Jading, I Gódor, G Auer, L Van der Perre, Challenges and enabling technologies for energy aware mobile radio networks. IEEE Commun. Mag. 48(11), 66-72 (2010)
9. L Saker, SE Elayoubi, T Chahed, Minimizing energy consumption via sleep mode in green base station. in IEEE Wireless Communications and Networking Conference (WCNC) 2010 (Sydney, Australia, 2010), pp. 1-6

10. E Oh, B Krishnamachari, X Liu, Z Niu, Toward dynamic energy-efficient operation of cellular network infrastructure. IEEE Commun. Mag. 49(6), 56-61 (2011)

11. Z Niu, Y Wu, J Gong, Z Yang, Cell zooming for cost-efficient Green cellular networks. IEEE Commun. Mag. 48(11), 74-79 (2011)

12. L Saker, SE Elayoubi, Sleep mode implementation issues in green base stations. in 21st Annual IEEE International Symposium on Personal, Indoor and Mobile Radio Communications (PIMRC) (Istanbul, Turkey, 2010), pp. 1681-1686

13. M Deruyck, E Tanghe, W Joseph, L Martens, Modelling and optimization of power consumption in wireless access networks. Elsevier Comput. Commun. 34(17), 2036-2046 (2011)

14. FH Raab, P Asbeck, S Cripps, PB Kenington, ZB Popovic, N Pothecary, JF Sevic, NO Sokal, RF and microwave power amplifier and transmitter technologies - part 1. High Freq. Electron, 22-36 (2003)

15. W Joseph, L Verloock, Influence of mobile phone traffic on base station exposure of the general public. Health Phys. 99(5), 631-638 (2010)

16. 3GPP, LTE: 3rd Generation Partnership Project: Technical Specification Group Radio Access Network: Evolved Universal Terrestrial Radio Access (E-UTRA): User Equipment (UE) radio transmission and reception (TS 36.101 v9.1.0 Release 9) (2009)

17. 3GPP, 3rd Generation Partnership Project: Technical Specification Group Radio Access Network: Physical layer aspects of UTRA High Speed Downlink Packet Access (Release 4), TR 25.848 v4.0.0 (2001)

doi:10.1186/1687-1499-2012-248

Cite this article as: Deruyck et al:: Characterization and optimization of the power consumption in wireless access networks by taking daily traffic variations into account. EURASIP Journal on Wireless Communications and Networking 2012 2012:248.

\section{Submit your manuscript to a SpringerOpen ${ }^{\mathcal{O}}$ journal and benefit from: \\ - Convenient online submission \\ - Rigorous peer review \\ - Immediate publication on acceptance \\ - Open access: articles freely available online \\ - High visibility within the field \\ - Retaining the copyright to your article}

Submit your next manuscript at $>$ springeropen.com 